\title{
Decision problems for 3-manifolds and their fundamental groups
}

\author{
MATTHiAs ASCHENBRENNER \\ STEFAN FRIEDL \\ HENRY WILTON
}

\begin{abstract}
We survey the status of some decision problems for 3-manifolds and their fundamental groups. This includes the classical decision problems for finitely presented groups (word problem, conjugacy problem, isomorphism problem), and also the homeomorphism problem for 3-manifolds and the membership problem for 3manifold groups.
\end{abstract}

$57 \mathrm{M} 05$

\section{Introduction}

The classical group-theoretic decision problems were formulated by Max Dehn in his work on the topology of surfaces [23] about a century ago. He considered the following questions about finite presentations $\langle A \mid R\rangle$ for a group $\pi$ :

(1) The word problem, which asks for an algorithm to determine whether a word on the generators $A$ represents the identity element of $\pi$.

(2) The conjugacy problem, which asks for an algorithm to determine whether two words on the generators $A$ represent conjugate elements of $\pi$.

(3) The isomorphism problem, which asks for an algorithm to determine whether two given finite presentations represent isomorphic groups.

Viewing $\pi$ as the fundamental group of a topological space (represented as a simplicial complex, say), these questions can be thought of as asking for algorithms to determine whether a given loop is null-homotopic, whether a given pair of loops is freely homotopic, and whether two aspherical spaces are homotopy-equivalent, respectively. We add some further questions that arise naturally: 
(4) The homeomorphism problem, which asks for an algorithm to determine whether two given triangulated manifolds are homeomorphic.

(5) The membership problem, where the goal is to determine whether a given element of a group lies in a specified subgroup.

Since the 1950s, it has been known that problems (1)-(5) have negative answers in the generality in which they have been formulated above. A finitely presented group with undecidable conjugacy problem was first constructed in 1954 by P S Novikov [82], and soon thereafter he [83] and W Boone [13] independently found a finitely presented group with undecidable word problem. (See [108] for an exposition.) Hence Problem (5), being a generalization of (1), is also undecidable. Similarly, Problem (3) is undecidable, since Adyan [2;1] and Rabin [93] showed that there is no algorithm for the more restrictive problem of determining whether a given finite presentation describes the trivial group. Problem (4) is undecidable even if we restrict to smooth manifolds of the same given dimension $\geq 4$; this was shown by Markov [65], as a corollary of the unsolvability of (3).

In contrast to this, in this paper we show that all these problems can now be solved for compact 3-manifolds and their fundamental groups, with the caveats that in (3) we restrict ourselves to closed, orientable 3-manifolds, and in (4) we restrict ourselves to orientable, irreducible 3-manifolds. Contrary to common perception (see [89, Section 7.1]) the homeomorphism problem for 3-manifolds is still open for reducible 3 -manifolds. We discuss the status of the homeomorphism problem in detail in Section 4.5.

The solutions to the first four problems, with the aforementioned caveats, have been known to the experts for a while, and as is to be expected, they all rely on the geometrization theorem. An algorithm for solving the membership problem was recently given in [28], and we will provide a summary of the main ideas in this paper. The solution to the membership problem requires not only the geometrization theorem but also the tameness theorem of Agol [3] and Calegari and Gabai [18], the virtually compact special theorem due to Agol [5] and Wise [114; 115; 116], as well as a result of Kapovich, Miasnikov and Weidmann [51].

Once a decision problem has been shown to be solvable, a natural next question concerns its complexity, and its implementability. The complexity of decision problems around 3 -manifolds is a fascinating topic which is not touched upon in the present paper, except for a few references to the literature here and there. Practical implementations of algorithms for 3-manifolds are discussed eg in [66].

The paper is organized as follows. In Section 1 we recall the precise statement of the (uniform) decision problems. In Section 2 we collect some basic definitions in group 
theory and 3-manifold topology. Then, we recall in Section 3 some of the key results in the study of 3-manifolds which appear time and again in the solutions to the decision problems. Finally in Section 4 we show that the five aforementioned problems are indeed solvable for (most) 3-manifolds and their fundamental groups. We conclude with a list of open problems in Section 5.

A final remark: the paper is written for group theorists and low-dimensional topologists alike. We hope that we succeeded in striking a balance between giving an exposition at an appropriate level of precision and conveying the main ideas behind the arguments. However, in order to make the paper more readable we refrained from giving some arguments in full detail.

Convention All 3-manifolds are assumed to be compact and connected. All surfaces in a 3-manifold are compact and properly embedded.

Acknowledgment The first author was partially supported by NSF grant number DMS-0969642. The second author is grateful to the organizers of the conference "Interactions between low dimensional topology and mapping class groups" at the Max-Planck Institute for Mathematics in Bonn for giving him an opportunity to speak. The second author was supported by the CRC "higher invariants" at the University of Regensburg. The third author was partially supported by an EPSRC Career Acceleration Fellowship. We wish to thank Benjamin Burton, David Futer and Greg Kuperberg for helpful comments. We are also very grateful for the detailed feedback provided by the referees.

\section{Decision problems}

To formulate group-theoretic decisions problems such as those from the introduction rigorously (and thus, to be able to prove their undecidability in general), one needs to make precise the informal notion of "algorithm."

\subsection{Turing machines}

Several mathematically rigorous definitions of algorithm were proposed in the 1930s, one of them being the notion of a Turing machine, defined by Turing in [111]. They were subsequently shown to all be equivalent in a natural sense. This is usually seen as evidence for the Church-Turing thesis, which asserts that every effective computation (be it on an abacus or a modern-day computer) can be carried out by a Turing machine. Hence in practice, to show the (mechanical) decidability of a mathematical question, 
one usually just sketches an algorithm informally and then appeals to the Church-Turing thesis to ascertain that it could in principle be translated into an actual Turing machine if so desired. In contrast, to prove undecidability of a given problem, one needs to resort to the precise definition of one's preferred model of computation. Since in this paper, we are mainly concerned with decidability results, below we only give a rough description of the structure and workings of a Turing machine; for a detailed and mathematically rigorous discussion, see [42].

A Turing machine is a hypothetical device consisting of a finite-length program as well as

(1) an infinite memory, usually visualized as a tape divided into infinitely many cells, sequentially ordered, where each cell can store exactly one symbol, either 0 or 1 , or be blank;

(2) a head that can read, write and delete symbols on the tape and move the tape left and right one (and only one) cell at a time; and

(3) a state register that stores the current state of the Turing machine, one of finitely many.

The Turing machine is provided with some input on the tape, with all but finitely many of the cells of the tape being initially blank. It then proceeds to modify this input according to its predetermined program, until it reaches a distinguished terminal state. If the machine never reaches this state, the computation will go on forever; but if it does (we say: if the Turing machine terminates), then the non-blank cells left on the tape are called the output of the Turing machine.

Turing machines can be viewed as accepting other objects as input and computing other objects as output, provided an encoding of these objects into finitely many finite strings of $0 \mathrm{~s}$ and $1 \mathrm{~s}$ has been chosen; these strings can then be written onto the tape of the Turing machine, separated by blanks, to provide the input for the machine. For example, Turing machines can work with words in the usual Latin alphabet, since its letters can be encoded by the binary representations of their ASCII codes. In fact, we may just as well use any countable, potentially infinite alphabet. More relevant for this paper, a finite presentation $\langle A \mid R\rangle$ of a group can be fed into a Turing machine by listing first an encoding of the elements of the generating set $A$ in binary representation, followed by the words in $R$, again suitably encoded into natural numbers via binary representation, and by separating these binary strings by blanks. Similarly one can also treat other finite mathematical objects, like finite simplicial complexes. Indeed, the (finite) data describing a Turing machine itself can be encoded (by a natural number) and thus be fed as an input to a Turing machine. A universal Turing machine is one that 
takes as input such an encoding of a Turing machine $T$, together with some input $s$, and then simulates the computation of $T$ with input $s$. In essence (except for the lack of infinitely large memory and the possibility of computational errors due to hardware failure), modern computers are implementations of universal Turing machines; see [41].

A decision problem is a subset of the set of possible inputs for a Turing machine. One says that a Turing machine solves a decision problem $S$ if it terminates for each possible input, and if it outputs "Yes" if the input belonged to $S$, and "No" otherwise. A decision problem is solvable if there exists a Turing machine solving it. Since there are only countably many Turing machines, but uncountable many decision problems, it is clear that there are many unsolvable decision problems. Using the concept of universal Turing machine, Turing [111] first exhibited a particular unsolvable decision problem, the halting problem: there is no Turing machine which takes as input an encoding of a Turing machine $T$ and decides whether $T$ halts upon input of the empty tape (with all cells blank). See [89] for a survey of many naturally occurring decision problems in mathematics which turned out to be undecidable.

A set $S$ of possible outputs of a Turing machine is called recursively enumerable if there exists a Turing machine, possibly non-terminating, that prints the elements of $S$, separated by blanks, onto its tape. Here we allow the possibility of repetitions, but it is easy to see that this notion of "recursively enumerable" does not change if we insist that the Turing machine writes each element of $S$ exactly once.

\subsection{Group-theoretical decision problems}

We refer to [72] for an authoritative survey of decision problems in group theory. These come in two flavors. The word problem, the conjugacy problem and the subgroup membership problem are all local, in the sense that they concern the relations of collections of elements of a fixed given finitely presented group. In contrast, the isomorphism problem is an example of a global decision problem, in the sense that it concerns relations between groups themselves.

1.2.1 Local decision problems We start with the local problems that we will consider. Fix a countable infinite alphabet $\mathcal{A}$ and a subset $A \subseteq \mathcal{A}$; below, $A$ usually is finite. All presentations of groups considered below are assumed to have the form $\langle A \mid R\rangle$ where $A \subseteq \mathcal{A}$. By a word on $A$ we mean an element of the free group $F(A)$ generated by $A$; that is, a formal expression of the form $a_{1}^{\epsilon_{1}} a_{2}^{\epsilon_{2}} \cdots a_{n}^{\epsilon_{n}}$, where $a_{1}, \ldots, a_{n} \in A$ and $\epsilon_{1}, \ldots, \epsilon_{n} \in\{ \pm 1\}$. If $A$ is a generating set for a group $\pi$, then there is a surjective group morphism $F(A) \rightarrow \pi$ with $a \mapsto a$ for each $a \in A$; given an element $g$ of $\pi$, every word $w \in F(A)$ which maps to $g$ under this morphism is said to represent the element $g$ of $\pi$. 
We now give more precise formulations of Dehn's decidability questions stated in the beginning of the introduction. Throughout, we assume that a fixed encoding of finite presentations $\langle A \mid R\rangle$ of groups and of finite collections of words from $F(\mathcal{A})$ as inputs for Turing machines has been chosen once and for all.

Definition 1.1 Let $\pi$ be a group with finite generating set $A$.

(1) The word problem in $\pi$ asks for an algorithm that takes as input a word $w$ on $A$ and determines whether $w$ represents the identity element of $\pi$. Note that whether the word problem for $\pi$ is solvable does not depend on the choice of the (finite) generating set $A$ for $\pi$.

(2) The conjugacy problem in $\pi$ asks for an algorithm that takes as input two words $w_{1}, w_{2} \in F(A)$ and determines whether $w_{1}$ and $w_{2}$ represent conjugate elements of $\pi$. Again, this notion is invariant under changing the (finite) generating set $A$.

(3) The membership problem in $\pi$ asks for an algorithm that takes as input words $v, w_{1}, \ldots, w_{k}$ on $A$ and determines whether $v$ represents an element of the subgroup $\left\langle g_{1}, \ldots, g_{k}\right\rangle$ of $\pi$ generated by the elements $g_{1}, \ldots, g_{k}$ represented by $w_{1}, \ldots, w_{k}$, respectively.

These "local" decision problems also have uniform analogues:

Definition 1.2 Let $\mathcal{G}$ be a class of finitely presentable groups.

(1) The word problem in $\mathcal{G}$ asks for an algorithm that takes as input a pair consisting of a presentation $\langle A \mid R\rangle$ of a group $\pi$ in $\mathcal{G}$ and a word $w \in F(A)$ and determines whether $w$ represents the identity element of $\pi$.

(2) The conjugacy problem in $\mathcal{G}$ asks for an algorithm that takes as input a presentation $\langle A \mid R\rangle$ of a group $\pi$ in $\mathcal{G}$ and two words $w_{1}, w_{2} \in F(A)$ and determines whether $w_{1}$ and $w_{2}$ represent conjugate elements of $\pi$.

(3) The membership problem in $\mathcal{G}$ asks for an algorithm that takes as input a presentation $\langle A \mid R\rangle$ of a group $\pi$ in $\mathcal{G}$ and words $v, w_{1}, \ldots, w_{k} \in F(A)$, and determines whether $v$ represents an element of the subgroup $\left\langle g_{1}, \ldots, g_{k}\right\rangle$ of $\pi$ generated by the elements $g_{1}, \ldots, g_{k}$ of $\pi$ represented by $w_{1}, \ldots, w_{k}$, respectively.

We say that the word problem is uniformly solvable in $\mathcal{G}$ if there is an algorithm which solves the word problem in $\mathcal{G}$; similarly for the other two decision problems. 
Note that in the definition above, we do not assume about $\mathcal{G}$ that there is an algorithm to decide whether a given finite presentation describes a group in $\mathcal{G}$. Although the class of 3-manifold groups is not algorithmically recognizable (see Lemma 3.4 below), for 3-manifold groups, all the algorithms we consider in this paper turn out to be uniform, in the sense that there is a single algorithm that solves the problem in question for any 3-manifold group, with its presentation provided as additional input for the algorithm.

1.2.2 Global decision problems The main global group-theoretic decision problem that we consider is the isomorphism problem: Consider a class $\mathcal{G}$ of finitely presentable groups, closed under isomorphism. The isomorphism problem in $\mathcal{G}$ asks for an algorithm that takes as input finite subsets $A_{1}, A_{2} \subseteq \mathcal{A}, R_{1} \subseteq F\left(A_{1}\right)$ and $R_{2} \subseteq F\left(A_{2}\right)$ such that the groups $\pi_{i}:=\left\langle A_{i} \mid R_{i}\right\rangle$ are in $\mathcal{G}$, and determines whether $\pi_{1}$ and $\pi_{2}$ are isomorphic. The next lemma shows that if we have determined that $\pi_{1}$ and $\pi_{2}$ are indeed isomorphic, then an isomorphism between them can be found algorithmically. Note that a group morphism $F(A) \rightarrow F\left(A^{\prime}\right)$ is uniquely specified by the images of the generators $a \in A$ of $F(A)$, and thus can be encoded as the output of a Turing machine.

Lemma 1.3 There exists an algorithm which, given finite presentations $\langle A \mid R\rangle$ and $\left\langle A^{\prime} \mid R^{\prime}\right\rangle$ of isomorphic groups $\pi, \pi^{\prime}$ as input, constructs a group morphism $F(A) \rightarrow F\left(A^{\prime}\right)$ which induces an isomorphism $\pi \rightarrow \pi^{\prime}$.

Proof We denote by $N$ the subgroup of $F(A)$ normally generated by $R$; thus $\pi \cong F(A) / N$. Similarly we define $N^{\prime}$. Note that group morphisms $\varphi: F(A) \rightarrow F\left(A^{\prime}\right)$ and $\varphi^{\prime}: F\left(A^{\prime}\right) \rightarrow F(A)$ gives rise to group morphisms $\pi \rightarrow \pi^{\prime}$ and $\pi^{\prime} \rightarrow \pi$ which are mutually inverse if and only if the following two conditions hold:

(1) $\varphi(g) \in N^{\prime}$ for all $g \in R$ and $\varphi^{\prime}\left(g^{\prime}\right) \in N$ for all $g^{\prime} \in R^{\prime}$.

(2) $\varphi^{\prime}(\varphi(a)) a^{-1} \in N$ for all $a \in A$ and $\varphi\left(\varphi^{\prime}\left(a^{\prime}\right)\right)\left(a^{\prime}\right)^{-1} \in N^{\prime}$ for all $a^{\prime} \in A^{\prime}$.

We now simultaneously enumerate all words in $N$ and $N^{\prime}$ as well as all group morphisms $\varphi: F(A) \rightarrow F\left(A^{\prime}\right)$ and $\varphi^{\prime}: F\left(A^{\prime}\right) \rightarrow F(A)$, and check whether conditions (1) and (2) are satisfied. By assumption there exists an isomorphism $\pi \rightarrow \pi^{\prime}$, and so after finitely many steps we will find a pair $\varphi, \varphi^{\prime}$ as above, satisfying (1) and (2).

A similar argument as in the previous proof shows that we may let $\langle A \mid R\rangle$ and $\left\langle A^{\prime} \mid R^{\prime}\right\rangle$ range over a recursively enumerable set of finite presentations:

Lemma 1.4 Let $\mathcal{R}, \mathcal{R}^{\prime}$ be recursively enumerable sets of finite presentations for groups, and suppose some group represented by an element of $\mathcal{R}$ is isomorphic to some group represented by an element of $\mathcal{R}^{\prime}$. Then there exists an algorithm which finds presentations $\langle A \mid R\rangle$ in $\mathcal{R}$ and $\left\langle A^{\prime} \mid R^{\prime}\right\rangle$ in $\mathcal{R}^{\prime}$ for groups $\pi$ and $\pi^{\prime}$, respectively, and also an isomorphism $\pi \rightarrow \pi^{\prime}$. 
Proof We simultaneously enumerate all presentations $\langle A \mid R\rangle$ in $\mathcal{R}$ and $\left\langle A^{\prime} \mid R^{\prime}\right\rangle$ in $\mathcal{R}^{\prime}$, all elements of the normal closure $N$ of $R$ in $F(A)$ and all elements of the normal closure $N^{\prime}$ of $R^{\prime}$ in $F\left(A^{\prime}\right)$, as well as all group morphisms $\varphi: F(A) \rightarrow F\left(A^{\prime}\right)$ and $\varphi^{\prime}: F\left(A^{\prime}\right) \rightarrow F(A)$, and check whether conditions (1) and (2) in the proof of Lemma 1.3 are satisfied.

The following lemma is used in Section 4.3.

Lemma 1.5 There exists an algorithm which upon input of finite presentations of groups $\Gamma$ and $\pi$, finitely many elements of $\pi$ generating a finite index subgroup $\pi_{0}$ of $\pi$, and a group morphism $\varphi: \Gamma \rightarrow \pi$, produces a finite presentation for $\Gamma_{0}:=\varphi^{-1}\left(\pi_{0}\right)$ and a set of coset representatives for $\Gamma_{0}$ in $\Gamma$.

Proof By going through all epimorphisms from $\pi$ to finite groups we first find a surjective group morphism $\psi: \pi \rightarrow G$ to a finite group $G$ and a subgroup $G_{0}$ of $G$ such that $\pi_{0}=\psi^{-1}\left(G_{0}\right)$. Replacing $\pi, \pi_{0}, \varphi$ by $G, G_{0}, \psi \circ \varphi$, respectively, we can thus assume that $\pi$ is finite. We can compute $\varphi(\Gamma)$ and $\varphi\left(\Gamma_{0}\right)=\pi_{0} \cap \varphi(\Gamma)$, and find coset representatives for $\varphi\left(\Gamma_{0}\right) \leq \varphi(\Gamma)$. We then compute preimages of these coset representatives under the map $\varphi$, which are then coset representatives for $\Gamma_{0} \leq \Gamma$. Using these coset representatives, the Reidemeister-Schreier process [61, Chapter II, Proposition 4.1] allows us to find a finite presentation for $\Gamma_{0}$.

The homeomorphism problem is a topological analogue of the isomorphism problem. For this, consider a class $\mathcal{M}$ of compact, triangulable manifolds. The homeomorphism problem in $\mathcal{M}$ asks for an algorithm that takes as input triangulations for two manifolds $M_{1}, M_{2} \in \mathcal{M}$ and determines whether or not $M_{1}$ and $M_{2}$ are homeomorphic.

\section{Basic definitions of group theory and 3-manifold topology}

As mentioned in the introduction, the intended audience for this paper consists of both group theorists and 3-manifold topologists. In order not to clutter the paper with definitions which are obvious to many, but perhaps not all, readers, in this section we summarize some of the basic definitions in 3-manifold topology and group theory which come up later in the paper.

\subsection{Special classes of 3-manifolds}

We start out with the definition of special classes of 3-manifolds, which form the "building blocks" of arbitrary 3-manifolds (see Section 3 below). 
First of all, a spherical 3-manifold is the quotient of $S^{3}$ by a finite subgroup $\Gamma$ of $\mathrm{SO}(4)$ acting freely by rotations on $S^{3}$; the fundamental group of such a spherical 3-manifold is isomorphic to $\Gamma$. Examples of spherical 3-manifolds are given by the Poincaré homology sphere and by lens spaces. Recall that given coprime integers $p, q \geq 1$ the corresponding lens space $L(p, q)$ is defined as

$$
L(p, q):=S^{3} /(\mathbb{Z} / p \mathbb{Z})=\left\{(x, y) \in \mathbb{C}^{2}:|x|^{2}+|y|^{2}=1\right\} /(\mathbb{Z} / p \mathbb{Z}),
$$

where $k+p \mathbb{Z} \in \mathbb{Z} / p \mathbb{Z}(k \in \mathbb{Z})$ acts on $S^{3}$ by

$$
(x, y) \mapsto\left(x e^{2 \pi i k / p}, y e^{2 \pi i k q / p}\right) .
$$

The fundamental group of $L(p, q)$ is isomorphic to $\mathbb{Z} / p \mathbb{Z}$.

A Seifert fibered manifold is a 3-manifold $N$ together with a decomposition into disjoint simple closed curves (called Seifert fibers) such that each Seifert fiber has a tubular neighborhood that forms a standard fibered torus. The standard fibered torus corresponding to a pair of coprime integers $(a, b)$ with $a \geq 1$ is the surface bundle of the automorphism of a disk given by rotation by an angle of $2 \pi b / a$, equipped with the natural fibering by circles. Every spherical 3-manifold is a Seifert fibered space (see [100]). Further examples of Seifert fibered spaces are given by Nil-manifolds, which are by definition 3-manifolds that are finitely covered by a torus bundle over $S^{1}$ whose monodromy action on the first homology of the torus is represented by an upper triangular matrix which is not diagonal.

A Seifert fibered space is called small if the base orbifold is a sphere with at most three cone points.

Later on we will also consider Sol-manifolds; these are 3-manifolds which are finitely covered by a torus bundle over $S^{1}$ such that the monodromy has real eigenvalues $\lambda, \lambda^{-1}$ with $\lambda>1$. Sol-manifolds are not Seifert fibered.

The other building blocks for 3-manifolds are hyperbolic 3-manifolds; these are the 3 -manifolds whose interior admits a complete metric of constant negative curvature -1 .

Thurston showed that, up to a certain equivalence, there exist precisely eight $3-$ dimensional geometries that model compact 3-manifolds. These geometries are: the 3-sphere with the standard spherical metric, Euclidean 3-space, hyperbolic 3-space, $S^{2} \times \mathbb{R}, \mathbb{H}^{2} \times \mathbb{R}$, the universal cover $\widehat{\operatorname{SL}(2, \mathbb{R})}$ of $\operatorname{SL}(2, \mathbb{R})$, and two further geometries called Nil and Sol. We refer to [99] for details. A 3-manifold is called geometric if it is an $X$-manifold for some geometry $X$. By [99] a 3-manifold is geometric if and only if it is either a Sol-manifold, or hyperbolic or Seifert fibered. 


\subsection{Combining 3-manifolds}

Given oriented 3-manifolds $M$ and $N$ we denote the connected sum of $M$ and $N$ by $M \# N$. Let $N$ be a 3-manifold. We say that $N$ is prime if $N$ cannot be written as a non-trivial connected sum of two manifolds, ie if $N \cong N_{1} \# N_{2}$, then


identify homeomorphic 3-manifolds.) Moreover, $N$ is called irreducible if every embedded $S^{2}$ bounds a 3-ball. Every irreducible 3-manifold is prime. Also, if $N$ is an orientable prime 3 -manifold with no spherical boundary components, then by [39, Lemma 3.13] either $N$ is irreducible or $N=S^{1} \times S^{2}$.

Recall that in this paper, all surfaces in 3-manifolds are assumed to be compact and properly embedded. A connected surface $\Sigma$ in a 3-manifold $N$ is called incompressible if the inclusion induced group morphism $\pi_{1} \Sigma \rightarrow \pi_{1} N$ is injective. A Haken manifold is an orientable and irreducible 3-manifold which contains an incompressible, orientable surface $\Sigma$ not homeomorphic to $D^{2}$ or $S^{2}$.

Finally, given a property $\mathcal{P}$ of manifolds we say that a 3 -manifold is virtually $\mathcal{P}$ if it admits a (not necessarily regular) finite cover which has the property $\mathcal{P}$.

\subsection{Definitions from group theory}

Let $\mathcal{P}$ be a property of groups and $\pi$ be a group. As for manifolds, we say that $\pi$ is virtually $\mathcal{P}$ if $\pi$ admits a (not necessarily normal) subgroup of finite index that satisfies $\mathcal{P}$. Furthermore, we say that $\pi$ is residually $\mathcal{P}$ if given any $g \in \pi$ with $g \neq 1$ there exists a morphism $\alpha: \pi \rightarrow G$ onto a group $G$ that satisfies $\mathcal{P}$ such that $\alpha(g) \neq 1$. A case of particular importance is when $\mathcal{P}$ is the class of finite groups, in which case $\pi$ is said to be residually finite.

We say that a subset $S$ of $\pi$ is separable if for any $g \in \pi \backslash S$, there exists a morphism $\alpha: \pi \rightarrow G$ to a finite group with $\alpha(g) \notin \alpha(S)$. We say that $\pi$ is locally extended residually finite (LERF) (or subgroup separable) if any finitely generated subgroup of $\pi$ is separable in this sense. Likewise, we say that $\pi$ is conjugacy separable if every conjugacy class in $\pi$ is separable.

Finally, let $\Gamma$ be a subgroup of $\pi$. We say that $\Gamma$ is a retract (of $\pi$ ) if there exists a retraction of $\pi$ onto $\Gamma$, that is, a group morphism $\pi \rightarrow \Gamma$ which is the identity on $\Gamma$. We say that $\Gamma$ is a virtual retract if there exists a finite index subgroup of $\pi$ which contains $\Gamma$ as a retract. 


\section{A quick trip through 3-manifold topology}

In this section we recall some of the key results in 3-manifold topology. Along the way we draw some first conclusions for the algorithmic study of 3-manifolds.

\subsection{Moise's theorem}

The first theorem of this section is a consequence of [75; 76]. We refer to [8] for precise references. The theorem says in particular that in the classification of 3-manifolds it does not matter whether we work in the category of topological, triangulable or smooth manifolds.

\section{Theorem 3.1 (Moise) Let $N$ be a topological 3-manifold.}

- $\quad N$ admits a finite triangulation, ie $N$ is homeomorphic to a finite simplicial complex; any two triangulations are related by a finite sequence of subdivisions and isotopies.

- $\quad N$ admits a smooth structure; any two smooth structures give rise to diffeomorphic manifolds.

We list various consequences of this theorem:

Corollary 3.2 The set of finite simplicial complexes which are homeomorphic to closed 3-manifolds is recursively enumerable. Furthermore, the set of finite simplicial complexes which are homeomorphic to closed orientable 3-manifolds is recursively enumerable.

Proof The first statement follows immediately from Moise's theorem and the fact that there is an algorithm to determine whether a given finite simplicial complex represents a 3-manifold. The algorithm in question simply checks that the link of each vertex is a 2-sphere. The details are left to the reader. (See the proof of [30, Lemma 5.4].) The second statement is proved in exactly the same way, except that now we also compute the third homology group to check for orientability.

It is clear that, given a triangulation of a 3-manifold, we may write down a finite presentation for its fundamental group, coming from the 2 -skeleton. Hence the previous corollary implies that there is a recursively enumerable set of finite presentations of 3-manifold groups which contains a presentation of each 3-manifold group. The next result shows that we can also pass from groups to (closed) 3-manifolds. 
Lemma 3.3 There is an algorithm that takes as input a finite presentation for a group $\pi$ and outputs a closed 3 -manifold $N$ with $\pi_{1} N \cong \pi$, if one exists.

Proof By Corollary 3.2 and the remark following it, the set of triangulations of closed 3-manifolds is recursively enumerable, and from a triangulation for a closed 3-manifold $N$, a finite presentation for $\pi_{1} N$ can be computed. Hence the claim follows from the argument used in the proof of Lemma 1.4. (The algorithm will not terminate if it is fed a finite presentation of a group that is not a fundamental group of a closed 3-manifold.)

In contrast to the previous lemma, we have:

Lemma 3.4 There is no algorithm that takes as input a finite presentation for a group $\pi$ and decides whether $\pi$ is the fundamental group of a closed 3-manifold.

This is an immediate consequence of the fact that although there is no algorithm to decide whether a given finite presentation describes the trivial group $[1 ; 2 ; 93]$, there is such an algorithm for the class of 3-manifold groups (see Corollary 4.9 below). One can also show Lemma 3.4 by observing that being a 3-manifold group is a "Markov property" in the sense of [93], using the existence of finitely presentable groups which cannot appear as subgroups of 3-manifold groups (for example, the well-known Baumslag-Solitar groups [9], or $\mathbb{Z}^{4}$ ).

In fact, the word problem is the only obstacle in the way of algorithmically recognizing closed geometric 3-manifolds:

Theorem 3.5 (Groves, Manning and Wilton [30]) Let $\mathcal{G}$ be any class of finitely presentable groups with uniformly solvable word problem. Then there is an algorithm which takes as input a finite presentation of a group from $\mathcal{G}$ and decides whether the group presented by it is the fundamental group of a closed geometric 3-manifold.

In a forthcoming paper, Groves, Manning and Wilton will prove the corresponding theorem in the remaining, non-geometric, case. So for example, one can determine algorithmically whether a residually finite finitely presented group is a 3-manifold group (cf Lemma 4.3 below).

Finally we observe that given the fundamental group $\pi$ of a 3-manifold, we may find a closed 3-manifold whose fundamental group retracts onto $\pi$.

Lemma 3.6 There is an algorithm that takes as input a finite presentation for the fundamental group $\pi$ of a 3-manifold and outputs a triangulation for a closed 3manifold $N$ together with an inclusion $\pi \hookrightarrow \pi_{1} N$ and a retraction $\pi_{1} N \rightarrow \pi$. 
Proof Let $M$ be a 3-manifold, and let $N$ be the double of $M$. Then $N$ is closed; note that $N$ is orientable if and only if $M$ is orientable. The "folding map" $N \rightarrow M$ is a retraction onto $M$, hence induces a left inverse $\pi_{1} N \rightarrow \pi_{1} M$ to the morphism $\pi_{1} M \rightarrow \pi_{1} N$ induced by inclusion.

The algorithm enumerates all closed 3-manifolds $N$ and all group morphisms $i: \pi \rightarrow$ $\pi_{1} N$ and $\rho: \pi_{1} N \rightarrow \pi$ satisfying $\rho \circ i=\mathrm{id}_{\pi}$. By the above discussion such $N, i$ and $\rho$ exist. An algorithm as in the proof of Lemma 1.3 will eventually find them.

A similar argument as in the proof of the previous lemma, letting $N$ run through all closed orientable 3-manifolds instead of all closed 3-manifolds, shows:

Lemma 3.7 There is an algorithm that takes as input a finite presentation for the fundamental group $\pi$ of an orientable 3-manifold and outputs a triangulation for a closed orientable 3-manifold $N$ together with an inclusion $\pi \hookrightarrow \pi_{1} N$ and a retraction $\pi_{1} N \rightarrow \pi$.

\subsection{The prime decomposition theorem}

The following theorem allows one to reduce many questions about 3-manifolds to the case of prime 3-manifolds. The existence of a prime decomposition is due to Kneser [55], and its uniqueness is due to Milnor [73, Theorem 1].

Theorem 3.8 (Prime decomposition theorem) Let $N$ be an oriented 3-manifold with no spherical boundary components.

- There exists a decomposition $N \cong N_{1} \# \ldots \# N_{r}$ where the 3-manifolds $N_{1}$, $\ldots, N_{r}$ are oriented prime 3-manifolds.

- If $N \cong N_{1} \# \cdots \# N_{r}$ and $N \cong N_{1}^{\prime} \# \cdots \# N_{s}^{\prime}$ where the 3-manifolds $N_{i}$ and $N_{i}^{\prime}$ are oriented and prime, then $r=s$ and (after reordering) there exist orientationpreserving diffeomorphisms $N_{i} \rightarrow N_{i}^{\prime}$.

The following fact says that at least for closed 3-manifolds, the prime decomposition can be computed; this is due to Jaco and Rubinstein [47] and Jaco and Tollefson [49, Algorithm 7.1].

Theorem 3.9 There is an algorithm that takes as input a finite triangulation for a closed, orientable 3-manifold $N$ and outputs a finite list of triangulations for closed oriented 3-manifolds $N_{1}, \ldots, N_{r}$ with the property that $N_{1} \# \ldots \# N_{r}$ is the prime decomposition of $N$. 
Remark 3.10 The problem of determining whether an orientable 3-manifold is irreducible (or prime) is decidable in space polynomial in the size of the triangulation. This is implicit in the work of Jaco and Rubinstein [47] and it is also proved explicitly by Ivanov [44, Theorem 1].

The prime decomposition theorem implies that the fundamental group of any orientable 3-manifold with no spherical boundary component can be written as the free product of fundamental groups of prime 3-manifolds. The following theorem can be viewed as a converse.

Theorem 3.11 (Kneser conjecture) Let $N$ be a compact, orientable 3-manifold with incompressible boundary and $\Gamma_{1}, \Gamma_{2} \leq \pi_{1} N$ with $\pi_{1} N \cong \Gamma_{1} * \Gamma_{2}$. Then there exist compact, orientable 3-manifolds $N_{1}$ and $N_{2}$ with $\pi_{1} N_{i} \cong \Gamma_{i}$ for $i=1,2$ and $N \cong N_{1} \# N_{2}$.

The Kneser conjecture was first proved by Stallings [107] in the closed case, and by Heil [37, page 244] in the bounded case.

\subsection{The geometrization theorem}

The central result in 3-manifold topology is the geometrization theorem, which had been conjectured by Thurston [110] and proved by Perelman.

Theorem 3.12 (Geometrization theorem) Let $N$ be an orientable, irreducible 3manifold with empty or toroidal boundary. Then there exist disjointly embedded incompressible tori $T_{1}, \ldots, T_{k}$ such that each component of $N$ cut along $T_{1} \cup \cdots \cup T_{k}$ is hyperbolic or Seifert fibered. Any such collection of tori with a minimal number of components is unique up to isotopy.

Jaco and Shalen [48] and Johannson [50] independently showed that $N$ splits along tori into Seifert fibered pieces and "atoroidal" pieces; they furthermore showed uniqueness for a minimal collection of such tori. These atoroidal pieces were then shown by Perelman $[86 ; 87 ; 88]$ to be either small Seifert spaces or hyperbolic. The full details of Perelman's proof can be found in [77; 78].

The decomposition of $N$ along a minimal collection of tori as in the geometrization theorem is called the JSJ (Jaco-Shalen-Johannson) decomposition of $N$. The tori in question are called the $J S J$ tori of $N$ and the components obtained by cutting along the JSJ tori are referred to as the JSJ components of $N$. If all of the JSJ components are Seifert fibered, then one calls $N$ a graph manifold. 
Remark 3.13 The geometrization conjecture has also been formulated for non-orientable 3-manifolds; we refer to [12, Conjecture 4.1] for details. To the best of our knowledge this has not been fully proved yet.

Jaco and Tollefson [49] and also Jaco, Letscher and Rubinstein [45] showed how to compute JSJ decompositions:

Theorem 3.14 There is an algorithm that takes as input a finite triangulation for a closed, orientable, irreducible 3-manifold $N$ and outputs the JSJ decomposition of $N$.

A proof of the following theorem is provided by [49, Algorithm 8.1].

Theorem 3.15 There is an algorithm that takes as input a finite triangulation for a closed, orientable, irreducible 3-manifold $N$ and determines whether $N$ is Seifert fibered or not.

\subsection{Consequences of the geometrization theorem}

The subsequent theorem is a consequence of the geometrization theorem together with work of Leeb [59]; see also [10, Theorem 2.3] for the extension to the case of non-toroidal boundary.

Theorem 3.16 If $N$ is an aspherical, orientable 3-manifold which is not a closed graph manifold, then the interior of $N$ admits a complete, non-positively curved, Riemannian metric.

It is important to note that there are graph manifolds that are not non-positively curved. For example Sol- and Nil-manifolds are not non-positively curved. We refer to [16; 17] and [59] for more information.

The following theorem is a consequence of the geometrization theorem in combination with the Mostow-Prasad rigidity theorem, work of Waldhausen [112, Corollary 6.5], and Scott [100, Theorem 3.1] and classical work on spherical 3-manifolds.

Theorem 3.17 Let $N$ and $N^{\prime}$ be two orientable, closed, prime 3-manifolds and let $\varphi: \pi_{1} N \rightarrow \pi_{1} N^{\prime}$ be an isomorphism. Then the following hold:

- If $N$ and $N^{\prime}$ are not lens spaces, then $N$ and $N^{\prime}$ are homeomorphic.

- If $N$ and $N^{\prime}$ are not spherical, then there exists a homeomorphism which induces $\varphi$. 


\subsection{The virtually compact special theorem}

We now turn to the theorem which is arguably the most important result in 3-manifold topology since the proof of the geometrization conjecture. The statement was proved by Wise $[114 ; 115 ; 116]$ for hyperbolic 3 -manifolds with boundary and for closed hyperbolic 3-manifolds which admit a geometrically finite surface. The general case of closed hyperbolic 3-manifolds is due to Agol [5].

Theorem 3.18 (Virtually compact special theorem) The fundamental group of a hyperbolic 3-manifold is the fundamental group of a virtually special compact cube complex.

The definition of a "special compact cube complex" goes back to Haglund and Wise [32]. We will not repeat the definition here, but we will give a precise algebraic statement which is a consequence of the preceding theorem, and sufficient for our purposes.

In order to do so, we need one more definition. Given a graph $G$ with vertex set $V=\left\{v_{1}, \ldots, v_{k}\right\}$ and edge set $E$, the corresponding right-angled Artin group is defined as

$$
\left.\Gamma=\Gamma(G)=\left\langle v_{1}, \ldots, v_{k}\right|\left[v_{i}, v_{j}\right]=1 \text { if }\left(v_{i}, v_{j}\right) \in E\right\rangle .
$$

For example, free groups of finite rank and finitely generated free abelian groups are right-angled Artin groups. See [19] for a survey on this class of groups. The following theorem now follows from Theorem 3.18.

Theorem 3.19 Let $N$ be a hyperbolic 3-manifold. Then $\pi_{1} N$ is virtually a virtual retract of a right-angled Artin group. This means that there exists a finite index subgroup $\pi^{\prime}$ of $\pi_{1} N$, a finite-index subgroup $\Gamma$ of a right-angled Artin group, and an embedding $\pi^{\prime} \rightarrow \Gamma$ which has a left-inverse $\Gamma \rightarrow \pi^{\prime}$.

We refer to [8] for details. The virtually compact special theorem has many striking consequences; for example, it implies by Agol's theorem [4] (see also [27]) that any hyperbolic 3-manifold with empty or toroidal boundary is virtually fibered. Together with the tameness theorem of Agol [3] and Calegari and Gabai [18] and work of Haglund [31] one also obtains the following theorem.

Theorem 3.20 Let $N$ be a hyperbolic 3-manifold and $\Gamma$ be a finitely generated subgroup of $\pi:=\pi_{1} N$. Then one of the following holds:

- $\quad \Gamma$ is a virtual retract of $\pi$.

- There exists a finite index subgroup $\pi^{\prime}$ of $\pi$ which contains $\Gamma$ as a normal subgroup with $\pi^{\prime} / \Gamma \cong \mathbb{Z}$.

In either case, $\Gamma$ is a separable subgroup of $\pi$. 
In the theorem the former case corresponds to $\Gamma$ being "geometrically finite", whereas the latter corresponds to $\Gamma$ being "geometrically infinite". Here we again refer to our survey [8] for details and precise references. Theorem 3.20 implies in particular that the fundamental group of a hyperbolic 3-manifold is subgroup separable.

\section{Decision problems}

This section is organized as follows. For each decision problem, we start by stating the theorem that describes its solvability in maximum generality and by sketching a proof or giving all the relevant references. We then go on to give a brief sample of the literature on the problem and discuss variations.

\subsection{The word problem}

The first classical decision problem which was solved for 3-manifold groups was the word problem.

Theorem 4.1 The word problem is uniformly solvable in the class of fundamental groups of 3-manifolds.

It is conceptually easiest to prove the theorem by appealing to the following theorem, a consequence of the geometrization theorem and work of Hempel [40].

Theorem 4.2 The fundamental group of each 3-manifold is residually finite.

Theorem 4.1 is now an immediate consequence of the following well-known observation of Dyson [24] and Mostowski [79], which in essence goes back to McKinsey [67]; see [26].

Lemma 4.3 The word problem is uniformly solvable in the class of finitely presented residually finite groups.

Proof Let $\pi=\langle A \mid R\rangle$ be a finite presentation for a residually finite group and let $w$ be a word in $A$. Systematically enumerate all words which are products of conjugates of elements in $R$ and check whether $w$ is one of them, and simultaneously also enumerate all morphisms $\alpha: \pi \rightarrow G$ to finite groups $G$ and test whether the element $g$ of $\pi$ represented by $w$ is in the kernel of $\alpha$. If $w$ represents the identity element of $\pi$, then our first procedure eventually detects this; on the other hand, if $w$ represents an element $g \neq 1$ of $\pi$, then by residual finiteness there exists a morphism $\alpha: \pi \rightarrow G$ to a finite group with $\alpha(g) \neq 1$, and our second procedure will detect this after finitely many steps. 
Although this approach gives a clean and uniform solution to the word problem, it has the disadvantage that it gives a very poor upper bound for its computational complexity. Another approach, which gives much better estimates of the complexity of the algorithm involved, uses the notion of automaticity. We refer to [25] for the definition of an automatic group and for further details.

For our purposes, the key fact is that the elements of an automatic group can efficiently be put into a canonical form [25, Theorem 2.3.10]; in particular, the word problem is (efficiently) solvable in automatic groups.

Theorem 4.4 [25, Theorem 12.4.7] Let $N$ be an orientable 3-manifold such that no prime factor admits Nil or Sol geometry. Then $\pi_{1} N$ is automatic.

By another result of [25], there is an algorithm that finds automatic structures on groups, and it follows that this solution to the word problem is uniform.

One way to quantify the efficiency of this solution to the word problem uses the notion of the Dehn function of a group. Given a finite presentation $\langle A \mid R\rangle$ of a group $\pi$ and word $w \in F(A)$ that represents the identity element in $\pi$, the area of $w$ is defined to be the minimal $n$ such that

$$
w=\prod_{i=1}^{n} g_{i} r_{i}^{ \pm 1} g_{i}^{-1}, \quad \text { where } g_{i} \in F(A) \text { and } r_{i} \in R .
$$

The Dehn function $\delta_{\langle A \mid R\rangle}: \mathbb{N} \rightarrow \mathbb{N}$ of the presentation $\langle A \mid R\rangle$ is defined by

$$
\delta_{\langle A \mid R\rangle}(n)=\max \left\{\operatorname{Area}(w): l_{A}(w) \leq n, w={ }_{\pi} 1\right\},
$$

where $l_{A}(w)$ is the length of the word $w$ in $F(A)$; that is, $\delta(n)$ is the maximal area among all words of length at most $n$ in $F(A)$ which represent the identity element of $\pi$. Although this definition depends on the presentation $\langle A \mid R\rangle$ of $\pi$, it turns out that the growth type of $\delta_{\langle A \mid R\rangle}$ only depends on $\pi$, and so we will often write $\delta_{\pi}$ instead. The word problem in $\pi$ is solvable if and only if its Dehn function is computable (by a Turing machine). Roughly speaking, the Dehn function of $\pi$ measures the difficulty of solving the word problem in $\pi$.

Definition 4.5 The group $\pi$ is said to satisfy a linear (respectively sub-quadratic, quadratic, exponential) isoperimetric inequality if $\delta_{\pi}$ is bounded above by a linear (respectively sub-quadratic, quadratic, exponential) function.

The terminology refers to a beautiful filling theorem which, for a compact Riemannian manifold $N$, relates the growth type of $\delta_{\pi_{1} N}$ to solutions to Plateau's problem in the universal cover of $N$ (see [14]). 
The connection to automaticity is given by [25, Theorem 2.3.12]:

Theorem 4.6 Every automatic group satisfies a quadratic isoperimetric inequality.

The combination of Theorems 4.4 and 4.6 now gives the following corollary.

Corollary 4.7 Let $N$ be an orientable 3-manifold such that no prime factor admits Nil or Sol geometry. Then $\pi_{1} N$ satisfies a quadratic isoperimetric inequality.

This result is optimal, as demonstrated by the following consequence of the geometrization theorem and a theorem of Gromov [29, 2.3.F] (see also [84]).

Theorem 4.8 For an irreducible 3-manifold $N$, the following are equivalent:

- $\pi_{1} N$ satisfies a sub-quadratic isoperimetric inequality.

- $\pi_{1} N$ satisfies a linear isoperimetric inequality.

- $\quad N$ is hyperbolic.

The Nil and Sol cases are also known: Nil-manifolds have cubic Dehn functions and Sol manifolds have exponential Dehn functions; see Example 8.1.1 and Theorem 8.1.3 of [25], respectively.

A third approach to the word problem is provided by non-positive curvature: if $N$ admits a non-positively curved metric then, by the filling theorem alluded to above, $\pi_{1} N$ admits a quadratic isoperimetric inequality. By Theorem 3.16 this gives a solution to the word problem for a very large class of 3-manifolds.

We note some consequences of the solvability of the uniform word problem for 3manifold groups:

Corollary 4.9 There is an algorithm which upon input of a finite presentation of a 3 -manifold group $\pi$, decides whether $\pi$ is trivial, respectively abelian.

Proof A finitely generated group $G$ is trivial if and only if each generator is trivial, and $G$ is abelian if and only if each pair of generators commutes. Both conditions can be effectively verified using an algorithm for the word problem. 


\subsection{The conjugacy problem}

The second of Dehn's decision problems also has a positive solution for 3-manifold groups:

Theorem 4.10 The conjugacy problem is uniformly solvable in the class of fundamental groups of 3-manifolds.

Préaux, extending Sela's work on knot groups [102], proved that the conjugacy problem is solvable, first for the fundamental groups of orientable 3-manifolds [90], and then also for the fundamental groups of non-orientable 3-manifolds [91]. (Note that, in contrast to many other group properties, solvability of the conjugacy problem does not automatically pass to finite extensions.) Although he does not explicitly state it, Préaux's solution to the conjugacy problem for 3-manifold groups is uniform.

For orientable 3-manifolds we can also give a proof of this theorem which is analogous to the first proof in the previous section. More precisely, building on the virtually compact special theorem and work of Minasyan [74] it was shown in [35, Theorem 1.3] that the fundamental group of any orientable $3-$ manifold $N$ is conjugacy separable. This means that given any non-conjugate $g, h \in \pi_{1} N$ there exists a morphism $\alpha: \pi_{1} N \rightarrow G$ to a finite group $G$ such that $\alpha(g)$ and $\alpha(h)$ are non-conjugate. It now follows from an argument similar to the proof of Lemma 4.3 that the conjugacy problem is uniformly solvable for fundamental groups of orientable 3-manifolds.

As in the last section, if a 3-manifold $N$ admits a non-positively curved metric, then [15, Theorem III. Г.1.12] gives another solution to the conjugacy problem for $\pi_{1} N$. However, as the constants involved in this theorem depend on the non-positively curved metric on $N$, this does not a priori give a uniform solution.

Finally, the conjugacy problem is not known to be solvable for automatic groups [25, Open Question 2.5.8]. The conjugacy problem is known to be solvable for biautomatic groups, but it is unknown whether automatic 3-manifold groups are biautomatic [8, Question 9.33].

\subsection{The membership problem}

The following theorem was recently proved in [28]. It should be contrasted with the fact that the membership problem is not solvable even for fairly simple groups like the direct product $F_{2} \times F_{2}$ of two copies of the free group $F_{2}$ on two generators [68; 70].

Theorem 4.11 The membership problem is uniformly solvable in the class of fundamental groups of 3-manifolds. 
In Theorem 3.20 we saw that fundamental groups of hyperbolic 3-manifolds are subgroup separable. Using the argument of the proof of Lemma 4.3 we then obtain a solution to the uniform membership problem for fundamental groups of hyperbolic 3manifolds. This approach works also for fundamental groups of Seifert fibered spaces, which are subgroup separable by work of Scott [98]. The argument can also be used for special classes of subgroups, eg subgroups carried by embedded surfaces [92], but this approach does not work in general. For example, Niblo and Wise [81, Theorem 4.2] showed that the fundamental groups of most graph manifolds are not subgroup separable. We thus see that we cannot hope to prove Theorem 4.11 in the general case by appealing to separability properties only.

In the following we will summarize the proof of Theorem 4.11. We refer to [28] for full details and for a careful discussion of the fact that we can give a uniform solution to the membership problem.

In the proof of Theorem 4.11 we employ the following useful lemma.

Lemma 4.12 Let $\mathcal{G}$ be a class of finitely presentable groups, and let $\mathcal{R}$ be a set of finite presentations of groups. Suppose that every group in $\mathcal{G}$ is virtually isomorphic to a group presented by some element of $\mathcal{R}$. If

- the membership problem is uniformly solvable in the class of groups presented by elements of $\mathcal{R}$, and

- $\mathcal{R}$ is recursively enumerable,

then the membership problem is also uniformly solvable in $\mathcal{G}$.

Proof Consider a group $\pi$ in $\mathcal{G}$. Using the Reidemeister-Schreier procedure, we may enumerate all subgroups of finite index in $\pi$. Because $\mathcal{R}$ is recursively enumerable, we will eventually find one, $\pi_{0}$ say, that we may confirm is in $\mathcal{R}$, using Lemma 1.4.

Let $H$ be a finitely generated subgroup of $\pi$, specified by a finite set of elements. By Lemma 1.5, we may compute a generating set for $H_{0}=H \cap \pi_{0}$ and a set of (left) coset representatives $h_{1}, \ldots, h_{k}$ for $H_{0}$ in $H$. Now, let $g \in \pi$ be given. For each $i$, we may determine whether $h_{i}^{-1} g \in \pi_{0}$, since membership in subgroups of finite index is always decidable. If there is no such $i$ then evidently $g \notin H$. Otherwise, if $h_{i}^{-1} g \in \pi_{0}$ then, using the solution to the membership problem in $\pi_{0}$, we may determine whether $h_{i}^{-1} g \in H_{0}$. Since $g \in H$ if and only if $h_{i}^{-1} g \in H_{0}$ for some $i$, this solves the membership problem in $\pi$.

Solvability of the membership problem in fundamental groups of graphs of groups was addressed by Kapovich, Miasnikov and Weidmann [51], who gave the following definition. See [7, Section 1.2] or [105] for the definition of a graph of groups. 
Definition 4.13 Consider a graph of finitely generated groups. In the following, $G_{v}$ is a vertex group and $G_{e}$ is an incident edge group. Such a graph of finitely generated groups is benign if:

(B1) For each vertex $v$ there is an algorithm to test membership of double cosets of the form $H G_{e}$ in $G_{v}$, where $e$ is an incident edge and $H$ is a finitely generated subgroup of $G_{v}$.

(B2) For each edge $e$ the group $G_{e}$ is slender, meaning that every subgroup of $G_{e}$ is finitely generated.

(B3) For each edge $e$ there is an algorithm to solve the membership problem in the edge group $G_{e}$.

(B4) For each vertex $v$ and incident edge $e$, there is an algorithm to compute generating sets for intersections $H \cap G_{e}$, where $H$ is a finitely generated subgroup of $G_{v}$.

Theorem 4.14 (Kapovich, Miasnikov and Weidmann [51, Theorem 5.13]) The membership problem is solvable for fundamental groups of benign graphs of groups in which every vertex group has solvable membership problem.

Remark 4.15 The given solution is not a priori uniform. However, it depends only on the graph of groups and the algorithms guaranteed by the hypotheses. If these can be found uniformly, then the solution to the membership problem is indeed uniform.

For future reference we record the following immediate corollary first proved by Mihaŭlova [69; 71]; see also [51, Corollary 5.16].

Corollary 4.16 Solvability of the membership problem is preserved under taking free products.

We now argue that the JSJ graph of groups of a closed 3-manifold is benign.

Condition (B1) follows from an argument as in Lemma 4.3 and the fact that given a fundamental group of a Seifert fibered space or a hyperbolic 3-manifold, any product of two finitely generated subgroups is separable. In the Seifert fibered case this was proved by Niblo [80] building on the aforementioned work of Scott [98]. In the hyperbolic case this is an extension of Theorem 3.20, which follows from work of Wise [116, Theorem 16.23] combined with work of Hruska [43, Corollary 1.6].

Condition (B2) is immediate, and (B3) follows from the fact that the membership problem in $\mathbb{Z}^{2}$ can be solved easily using basic algebra. 
Therefore, to prove the solvability of the membership problem in 3-manifold groups, it now remains to address (B4). We deal with the Seifert fibered and hyperbolic cases separately, in the following two lemmas.

Lemma 4.17 Let $N$ be a hyperbolic 3-manifold with toroidal boundary. Let $P$ be a cusp subgroup of $\pi=\pi_{1} N$. There is an algorithm, uniform in $\pi$ and $P$, that takes as input a finite set of elements that generate a subgroup $\Gamma$ of $\pi$, and computes a generating set for $\Gamma \cap P$.

Proof By Theorem 3.20, one of the following happens:

(1) There exists a finite-index subgroup $\pi_{0}$ of $\pi$ and a retraction $\rho: \pi_{0} \rightarrow \Gamma$.

(2) There exists a finite-index subgroup $\pi_{0}$ and a morphism $p: \pi_{0} \rightarrow \mathbb{Z}$ such that $\Gamma=\operatorname{Ker} p$.

We now run two algorithms simultaneously: A naive search using the ReidemeisterSchreier algorithm to find $\pi_{0}$ and $\rho$ as in Case (1), and a naive search using the Reidemeister-Schreier algorithm and the algorithm used in the proof of Lemma 1.4 looking for $\pi_{0}$ and $p$ as in Case (2). Since Case (1) or (2) hold, one of these algorithms will terminate. By Lemma 1.5, in either case we can compute generators for $P_{0}=$ $\pi_{0} \cap P$.

In Case (2), $\Gamma \cap P=(\operatorname{Ker} p) \cap P_{0}$, which can be computed by standard linear algebra.

Suppose that we are in Case (1). We note that $\Gamma \cap P=\rho\left(P_{0}\right) \cap P_{0}$. Using the solution to the word problem in $\pi$ we can determine whether all generators of $\rho\left(P_{0}\right)$ and $P_{0}$ commute, ie whether $\left[\rho\left(P_{0}\right), P_{0}\right]=1$.

First suppose that $\left[\rho\left(P_{0}\right), P_{0}\right]=1$. It follows from the well-known fact that $P_{0}$ is maximal abelian in $\pi_{0}$ (see [8, Theorem 3.1]) that then $\rho\left(P_{0}\right) \subseteq P_{0}$, which implies that $\Gamma \cap P=\rho\left(P_{0}\right)$.

Now suppose that $\left[\rho\left(P_{0}\right), P_{0}\right] \neq 1$. The fact that $N$ is hyperbolic implies by $[8$, Corollary 3.11] that the centralizer of any non-identity element in $\pi_{0}$ is abelian. It now follows that $\rho\left(P_{0}\right) \cap P_{0}=1$ and so $\Gamma \cap P=1$.

A similar argument deals with the case of a product manifold:

Lemma 4.18 Let $N$ be a compact Seifert-fibered manifold with boundary and let $P$ be a cusp subgroup of $\pi=\pi_{1} N$. There is an algorithm, uniform in $\pi$ and $P$, that takes as input a finite set of elements that generate a subgroup $\Gamma$ of $\pi$, and computes a generating set for $\Gamma \cap P$. 
Proof Since $N$ is finitely covered by a product $\Sigma \times S^{1}$ (where $\Sigma$ is a compact surface with boundary), one quickly reduces to the case $N=\Sigma \times S^{1}$ (see [28, Lemma 26] for full details).

The case that $\Sigma$ is a disk or an annulus is trivial. We therefore henceforth assume that $\chi(\Sigma)<0$. Note that, since every finitely generated subgroup of a surface group $\pi_{1} \Sigma$ is a virtual retract (this is implicit in [98]), it follows easily that every finitely generated subgroup of $\pi_{1} \Sigma \times \mathbb{Z}$ is a virtual retract. Therefore, a naive search using the Reidemeister-Schreier algorithm will find a finite-index subgroup $\pi_{0}$ of $\pi$ and a retraction $\rho: \pi_{0} \rightarrow \Gamma$. As in the proof of Lemma 4.17, we can compute generators for $P_{0}=\pi_{0} \cap P$.

Again, we note that $\Gamma \cap P=\rho\left(P_{0}\right) \cap P_{0}$. As before, an explicit computation again determines whether $\left[\rho\left(P_{0}\right), P_{0}\right]=1$. If so then, just as before, because $P_{0}$ is maximal abelian we have $\rho\left(P_{0}\right) \subseteq P_{0}$ and so $\rho\left(P_{0}\right)=\Gamma \cap P$. If not, then by the commutative transitivity of $\pi_{1} \Sigma$, we deduce that $\Gamma \cap P=\rho\left(P_{0}\right) \cap P_{0}$ is contained in the center $Z_{0}$ of $\pi_{0}$ and so it suffices to compute $\rho\left(P_{0}\right) \cap Z_{0}$. (Here recall that a group is commutative transitive if $[a, b]=1$ and $[b, c]=1$ imply that $[a, c]=1$.) But now $\rho\left(P_{0}\right) \cap Z_{0}$ can be seen in the abelianization of $\pi_{0}$, and so can be computed by elementary linear algebra.

We are now ready to prove that the membership problem is solvable.

Proof of Theorem 4.11 We start out by showing that it suffices to show that the membership problem is uniformly solvable for the class of fundamental groups of orientable 3-manifolds. To see this, let $\pi$ be the fundamental group of a 3-manifold $N$. Then $\pi^{\prime}:=\operatorname{Ker}\left(\pi \rightarrow H_{1}\left(\pi ; \mathbb{F}_{2}\right)\right)$ is the fundamental group of an orientable $3-$ manifold. By Lemma 1.5, we can determine a presentation for $\pi^{\prime}$. Moreover, by Lemma 4.12, a solution to the membership problem for $\pi^{\prime}$ also gives a solution to the membership problem for $\pi$. This concludes the proof of the claim.

By the claim and Lemma 3.7, we may now assume that $\pi$ is the fundamental group of a closed orientable 3-manifold $N$ and that we furthermore have a triangulation for $N$ available. By Theorem 3.9 we can determine the prime decomposition of $N$. By Corollary 4.16 it thus suffices to consider the prime components of $N$. We can henceforth assume that $N$ is irreducible.

Using Theorem 3.15 we determine whether $N$ is Seifert fibered. As mentioned before, in this case, $\pi$ is subgroup separable by [98]; this immediately gives a uniform solution to the membership problem. If $N$ is not Seifert fibered, then we use Theorem 3.14 to determine the JSJ decomposition of $N$. The corresponding graph of groups decomposition of $\pi$ is benign by Lemmas 4.17 and 4.18. So the result follows from Theorem 4.14. 
Remark 4.19 As a further consequence of [51, Theorem 5.8(b)], we also get that the uniform finite presentation problem for closed, orientable 3-manifold groups is solvable. That is, there is an algorithm that takes as input a finite set $S$ of elements of a fundamental group $\pi$ of a closed, orientable 3-manifold and outputs a finite presentation for the subgroup $\langle S\rangle$ of $\pi$, and this algorithm is uniform in $\pi$.

\subsection{The isomorphism problem}

Much of the material in this section derives from [103, Section 10]. We refer to [11] for further details.

Theorem 4.20 The isomorphism problem for the class of fundamental groups of closed, orientable 3-manifolds is solvable.

In the remainder of this section we will sketch the proof of Theorem 4.20. First, it follows from Lemma 3.3 together with Theorems 3.9 and 3.11 that it suffices to solve the isomorphism problem for fundamental groups of closed, orientable and irreducible 3-manifolds.

At this stage, the argument divides into the cases in which $N$ is Haken and $N$ is non-Haken. First, we need to be able to determine which case we are in. That we can follows from [46, Theorem 4.3].

Theorem 4.21 (Jaco and Oertel) There is an algorithm to determine whether a given closed, irreducible 3-manifold $N$ is Haken.

The Haken case is dealt with by the following theorem of Haken [34], Waldhausen [113], Hemion [38] and Matveev [66].

Theorem 4.22 (Haken) There is an algorithm that determines whether an input pair of orientable Haken 3-manifolds are homeomorphic.

By Theorem 3.17, this gives rise to a group-theoretic analogue.

Corollary 4.23 The isomorphism problem for the class of fundamental groups of closed, orientable, Haken 3-manifolds is solvable.

It remains to deal with the non-Haken case which, by geometrization, divides into three cases: spherical, small Seifert fibered with infinite fundamental group and hyperbolic. The next theorem asserts that these can be recognized algorithmically. 
Theorem 4.24 There is an algorithm to determine whether a given closed, orientable, non-Haken 3-manifold is spherical, small Seifert fibered with infinite fundamental group, or hyperbolic.

See [103, Theorem 10.5] for the proof. The main ingredient is Papasoglu's algorithm for finding word-hyperbolic structures on a group [85]. Alternatively, one could use Manning's algorithm [63].

It now remains to solve the isomorphism problem in these three cases. The isomorphism problem is easily solvable for finite groups: given finite presentations $\langle A \mid R\rangle$ and $\left\langle A^{\prime} \mid R^{\prime}\right\rangle$ for groups $\pi, \pi^{\prime}$, respectively, first use the Todd-Coxeter algorithm (see [106]) to construct multiplication tables for $\pi, \pi^{\prime}$, using which it is straightforward to determine whether $\pi \cong \pi^{\prime}$. The remaining two cases are dealt with by the following theorems of Sela.

Theorem 4.25 (Sela) The isomorphism problem is solvable for the infinite groups which are fundamental groups of small Seifert fibered manifolds.

The proof of this theorem is the content of [103, pages 280-281]. The problem quickly reduces to the isomorphism problem for triangle groups. Using more heavy machinery, one could instead invoke Dahmani and Guirardel's solution to the isomorphism problem for word-hyperbolic groups with torsion [22].

Theorem 4.26 (Sela) The isomorphism problem is solvable for fundamental groups of closed, orientable hyperbolic 3-manifolds.

This is an immediate corollary of the main theorem of [103]. Scott and Short [101] gave an alternative proof using Manning's algorithm [63].

This completes the proof of Theorem 4.20.

\subsection{The homeomorphism problem}

In several places in the literature, it is stated that an affirmative solution to the homeomorphism problem for all closed, orientable 3-manifolds follows from the geometrization theorem and the aforementioned work of Sela [103]. In fact, we have:

Theorem 4.27 The homeomorphism problem is solvable for orientable, irreducible 3-manifolds with only incompressible boundary components. 
Note that by Theorem 3.1, two 3-manifolds are diffeomorphic if and only if they are homeomorphic. A solution to the homeomorphism problem is thus also a solution to the Diffeomorphism problem.

Proof Let $N$ and $N^{\prime}$ be orientable, irreducible 3-manifolds. It is well-known how to compute the first Betti numbers $b_{1} N, b_{1} N^{\prime}$ of $N$ respectively $N^{\prime}$.

First assume that $b_{1} N \geq 1$ or $b_{1} N^{\prime} \geq 1$, say $b_{1} N \geq 1$. Evidently $N^{\prime}$ is homeomorphic to $N$ only if $b_{1} N^{\prime} \geq 1$, so we may also assume $b_{1} N^{\prime} \geq 1$. Since the boundary components of $N$ and $N^{\prime}$ are incompressible it follows from a standard argument (see [8, (C.18)]) that $N$ and $N^{\prime}$ are Haken. A solution to the homeomorphism problem for Haken manifolds has been given by Matveev [66, Theorem 6.1.1], building on earlier work of Hemion [38] and Haken [34].

We now suppose that $b_{1} N=b_{1} N^{\prime}=0$. Since $N$ is irreducible it follows that $N$ is either the 3-ball or closed, and similarly for $N^{\prime}$. Since connected closed orientable surfaces are classified by their Euler characteristics one can easily determine whether the boundaries of $N$ and $N^{\prime}$ are homeomorphic to $S^{2}$. Hence we can now restrict to the case that $N$ and $N^{\prime}$ are closed.

By Theorem 4.20 we can determine whether $\pi_{1} N$ and $\pi_{1} N^{\prime}$ are isomorphic. If they are, then Theorem 3.17 implies that $N$ and $N^{\prime}$ are either homeomorphic or they are both lens spaces. Suppose that $N$ and $N^{\prime}$ are lens spaces with fundamental group $\mathbb{Z} / p \mathbb{Z}$. By the classification of lens spaces we can provide a complete, necessarily finite, list of pairwise non-homeomorphic triangulated lens spaces $L_{1}, \ldots, L_{k}$ with fundamental group $\mathbb{Z} / p \mathbb{Z}$. Since $N$ and $N^{\prime}$ are PL-isomorphic to precisely one of the $L_{i}$ we can now determine whether or not $N$ and $N^{\prime}$ are homeomorphic. Alternatively, one can use Reidemeister's original approach [94] via the torsion invariant to determine whether $N$ and $N^{\prime}$ are homeomorphic.

We do not doubt that the hypotheses of Theorem 4.27 can be relaxed; in particular, most experts agree that a solution to the homeomorphism problem among closed, orientable 3-manifolds that are not necessarily irreducible is within the reach of current techniques. However, the details of such an algorithm do not, as far as we know, appear in the literature. $^{1}$

In order to deal with the reducible case, one needs to address the oriented nature of the uniqueness part of the Kneser-Milnor decomposition. Specifically, a proof of the following statement is required:

\footnotetext{
${ }^{1}$ While this paper was in publication, a detailed treatment of the oriented homeomorphism problem appeared [58].
} 
There is an algorithm that determines whether a closed, orientable, irreducible

3-manifold admits an orientation-reversing self-homeomorphism.

In the case of a hyperbolic manifold $M$ of finite volume, this fact can be deduced from published results. By Mostow rigidity, any automorphism of $\pi_{1} M$ is induced by a self-homeomorphism. The extension of Sela's results by Dahmani and Groves [20] makes it possible to list the elements $\left\{\alpha_{i}\right\}$ of the (finite) outer automorphism group of $\pi_{1} M$, and computing the action on $H_{3}$ determines whether or not each $\alpha_{i}$ reverses orientation. Alternatively, one can attempt to modify the Scott-Short algorithm [101] (cf [58]).

In order to complete the solution to the homeomorphism problem in the closed case, one needs to analyze the remaining Seifert fibered and Haken cases. Perhaps a variation of the Haken-Hemion-Matveev algorithm [66, Theorem 6.1.1] can be used to solve the oriented homeomorphism problem in the Haken case. Alternatively, using the canonical properties of the JSJ decomposition, it should be possible to reduce the question to the hyperbolic and Seifert fibered cases.

In our opinion, a detailed solution of the above problem would be a great service to the community, and fill an important gap in the literature.

Another problem that has attracted a lot of attention is the problem of deciding whether a given 3-manifold is homeomorphic to a particular kind of 3-manifold (eg spheres, handlebodies etc). For example, Rubinstein [96] gave an algorithm that determines whether a 3-manifold is homeomorphic to a 3-sphere, whose correctness was shown in [109]. Later, Ivanov [44] and Schleimer [97] showed that this problem is the complexity class NP, that is, decidable in nondeterministic polynomial time (in size of the triangulation). In his paper [44], Ivanov also gave another proof of the result of Hass, Lagarias and Pippenger [36] that the problem of detecting whether a knot (represented by a triangulation of its complement) is the unknot, which was first shown to be decidable by Haken [33], is in NP. Kuperberg [57], using a theorem of Koiran [56], has shown that modulo the generalized Riemann hypothesis, unknot detection is also in co-NP, so (assuming also standard conjectures in complexity theory) not NP-hard. For some NP-complete decision problems in knot theory, see [6].

\section{Open problems}

We conclude this survey paper with a list of problems and conjectures. As we saw in Theorem 4.20, the isomorphism problem for the class of fundamental groups of closed, orientable 3-manifolds has an algorithmic solution. It is natural to ask whether the restriction to closed 3-manifolds is necessary. 
Question 5.1 Is there an algorithm which determines whether two fundamental groups of 3-manifolds are isomorphic?

The equation problem asks for a solution to the problem whether any set of "equations" over a group has a solution. It generalizes both the word problem and the conjugacy problem, and has been solved for torsion-free hyperbolic groups by Makanin [62] and Rips and Sela [95], for hyperbolic groups with torsion by Dahmani and Guirardel [21] and for fundamental groups of Seifert fibered spaces by Liang [60]. The following question thus arises.

Question 5.2 Is the equation problem solvable for the fundamental group of any 3-manifold?

Even more ambitiously, one may ask about the decidability of the full elementary theory of each 3-manifold group viewed as a structure in the language of groups, in the sense of model theory; see [64, Example 1.2.5].

Question 5.3 Let $\pi$ be a 3-manifold group. Is the first-order theory of $\pi$ decidable?

See $[52 ; 54]$ for work on this problem in the case where $\pi$ is a free group respectively a torsion-free hyperbolic group, but see also [53; 104].

Finally, we return to topological decision problems. A 3-manifold pair is a pair $(N, S)$, where $N$ is a 3 -manifold and $S$ is a subsurface in $\partial N$. For example, let $L=L_{1} \cup \cdots \cup L_{n}$ be an oriented link in a 3-manifold $Y$. Pick a tubular neighborhood $\nu L$ in $Y$ and denote by $\mu_{1}, \ldots, \mu_{n}$ the meridians of $L_{1}, \ldots, L_{n}$, respectively. The diffeomorphism class of $(Y, L)$ is then determined by the diffeomorphism class of the 3-manifold pair $\left(Y \backslash v L, \mu_{1} \times I \cup \cdots \cup \mu_{n} \times I\right)$. Sutured manifolds give naturally rise to 3-manifold pairs.

Question 5.4 Is there a solution to the homeomorphism problem for 3-manifold pairs?

\section{References}

[1] S I Adyan, Finitely presented groups and algorithms, Dokl. Akad. Nauk SSSR 117 (1957) 9-12 MR0095873

[2] S I Adyan, Unsolvability of some algorithmic problems in the theory of groups, Trudy Moskov. Mat. Obšč. 6 (1957) 231-298 MR0095872

[3] I Agol, Tameness of hyperbolic 3-manifolds, preprint (2004) arXiv:math/0405568 
[4] I Agol, Criteria for virtual fibering, J. Topol. 1 (2008) 269-284 MR2399130

[5] I Agol, The virtual Haken conjecture, Doc. Math. 18 (2013) 1045-1087 MR3104553

[6] I Agol, J Hass, W Thurston, The computational complexity of knot genus and spanning area, Trans. Amer. Math. Soc. 358 (2006) 3821-3850 MR2219001

[7] M Aschenbrenner, S Friedl, 3-manifold groups are virtually residually $p$, Mem. Amer. Math. Soc. 1058, Amer. Math. Soc. (2013) MR3100378

[8] M Aschenbrenner, S Friedl, H Wilton, 3-manifold groups, EMS Series Lect. Math. 20, Euro. Math. Soc., Zürich (2015)

[9] G Baumslag, D Solitar, Some two-generator one-relator non-Hopfian groups, Bull. Amer. Math. Soc. 68 (1962) 199-201 MR0142635

[10] I Belegradek, Topology of open nonpositively curved manifolds, preprint (2014) arXiv: 1306.1256

[11] L Bessières, G Besson, M Boileau, S Maillot, J Porti, Geometrisation of 3-manifolds, EMS Tracts in Mathematics 13, Euro. Math. Soc., Zürich (2010)

[12] F Bonahon, Geometric structures on 3-manifolds, from: "Handbook of geometric topology", (R J Daverman, R B Sher, editors), North-Holland, Amsterdam (2002) 93164 MR1886669

[13] W W Boone, The word problem, Ann. of Math. 70 (1959) 207-265 MR0179237

[14] M R Bridson, The geometry of the word problem, from: "Invitations to geometry and topology”, (M R Bridson, S M Salamon, editors), Oxford Grad. Texts Math. 7, Oxford Univ. Press (2002) 29-91 MR1967746

[15] M R Bridson, A Haefliger, Metric spaces of non-positive curvature, Grundl. Math. Wissen. 319, Springer, Berlin (1999) MR1744486

[16] S V Buyalo, V L Kobel'skiǔ, Geometrization of graph-manifolds, I: Conformal geometrization, Algebra i Analiz 7 (1995) 1-45 MR1347511

[17] S V Buyalo, V L Kobel'skiü, Geometrization of graph-manifolds, II: Isometric geometrization, Algebra i Analiz 7 (1995) 96-117 MR1353491

[18] D Calegari, D Gabai, Shrinkwrapping and the taming of hyperbolic 3-manifolds, J. Amer. Math. Soc. 19 (2006) 385-446 MR2188131

[19] R Charney, An introduction to right-angled Artin groups, Geom. Dedicata 125 (2007) 141-158 MR2322545

[20] F Dahmani, D Groves, The isomorphism problem for toral relatively hyperbolic groups, Publ. Math. Inst. Hautes Études Sci. (2008) 211-290 MR2434694

[21] F Dahmani, V Guirardel, Foliations for solving equations in groups: Free, virtually free, and hyperbolic groups, J. Topol. 3 (2010) 343-404 MR2651364

[22] F Dahmani, V Guirardel, The isomorphism problem for all hyperbolic groups, Geom. Funct. Anal. 21 (2011) 223-300 MR2795509 
[23] M Dehn, Über unendliche diskontinuierliche Gruppen, Math. Ann. 71 (1911) 116-144 MR1511645

[24] V H Dyson, The word problem and residually finite groups, Not. Amer. Math. Soc. 11 (1964) 734

[25] D B A Epstein, J W Cannon, D F Holt, S V F Levy, MS Paterson, W P Thurston, Word processing in groups, Jones and Bartlett, Boston (1992) MR1161694

[26] T Evans, Some connections between residual finiteness, finite embeddability and the word problem, J. London Math. Soc. 1 (1969) 399-403 MR0249344

[27] S Friedl, T Kitayama, The virtual fibering theorem for 3-manifolds, Enseign. Math. 60 (2014) 79-107 MR3262436

[28] S Friedl, H Wilton, The membership problem for 3-manifold groups is solvable, preprint (2014) arXiv:1401.2648 to be published in Alg. Geom. Topol.

[29] M Gromov, Hyperbolic groups, from: "Essays in group theory", (S M Gersten, editor), Math. Sci. Res. Inst. Publ. 8, Springer, New York (1987) 75-263 MR919829

[30] D Groves, J F Manning, H Wilton, Recognizing geometric 3-manifold groups using the word problem, preprint (2012) arXiv:1210.2101

[31] F Haglund, Finite index subgroups of graph products, Geom. Dedicata 135 (2008) 167-209 MR2413337

[32] F Haglund, D T Wise, Special cube complexes, Geom. Funct. Anal. 17 (2008) 15511620 MR2377497

[33] W Haken, Theorie der Normalfächen, Acta Math. 105 (1961) 245-375 MR0141106

[34] W Haken, Über das Homöomorphieproblem der 3-Mannigfaltigkeiten, I, Math. Z. 80 (1962) 89-120 MR0160196

[35] E Hamilton, H Wilton, P A Zalesskii, Separability of double cosets and conjugacy classes in 3-manifold groups, J. Lond. Math. Soc. 87 (2013) 269-288 MR3022716

[36] J Hass, J C Lagarias, N Pippenger, The computational complexity of knot and link problems, J. ACM 46 (1999) 185-211 MR1693203

[37] W Heil, On Kneser's conjecture for bounded 3-manifolds, Proc. Cambridge Philos. Soc. 71 (1972) 243-246 MR0312503

[38] G Hemion, On the classification of homeomorphisms of 2-manifolds and the classification of 3-manifolds, Acta Math. 142 (1979) 123-155 MR512214

[39] J Hempel, 3-manifolds, Ann. of Math. Studies 86, Princeton Univ. Press (1976) MR0415619

[40] J Hempel, Residual finiteness for 3-manifolds, from: "Combinatorial group theory and topology”, (S M Gersten, J R Stallings, editors), Ann. of Math. Stud. 111, Princeton Univ. Press (1987) 379-396 MR895623 
[41] R Herken (editor), The universal Turing machine: A half-century survey, 2nd edition, Computerkultur 2, Springer, Berlin (1995) MR1362572

[42] J E Hopcroft, J D Ullman, Introduction to automata theory, languages, and computation, Addison-Wesley, Reading, MA (1979) MR645539

[43] G C Hruska, Relative hyperbolicity and relative quasiconvexity for countable groups, Algebr. Geom. Topol. 10 (2010) 1807-1856 MR2684983

[44] S V Ivanov, The computational complexity of basic decision problems in 3-dimensional topology, Geom. Dedicata 131 (2008) 1-26 MR2369189

[45] W Jaco, D Letscher, J H Rubinstein, Algorithms for essential surfaces in 3manifolds, from: "Topology and geometry: commemorating SISTAG”, (A J Berrick, M C Leung, X Xu, editors), Contemp. Math. 314, Amer. Math. Soc. (2002) 107-124 MR1941626

[46] W Jaco, U Oertel, An algorithm to decide if a 3-manifold is a Haken manifold, Topology 23 (1984) 195-209 MR744850

[47] W Jaco, J H Rubinstein, 0-efficient triangulations of 3-manifolds, J. Differential Geom. 65 (2003) 61-168 MR2057531

[48] W H Jaco, P B Shalen, Seifert fibered spaces in 3-manifolds, Mem. Amer. Math. Soc. 220, Amer. Math. Soc. (1979) MR539411

[49] W Jaco, J L Tollefson, Algorithms for the complete decomposition of a closed 3manifold, Illinois J. Math. 39 (1995) 358-406 MR1339832

[50] K Johannson, Homotopy equivalences of 3-manifolds with boundaries, Lecture Notes in Mathematics 761, Springer, Berlin (1979) MR551744

[51] I Kapovich, R Weidmann, A Miasnikov, Foldings, graphs of groups and the membership problem, Internat. J. Algebra Comput. 15 (2005) 95-128 MR2130178

[52] O Kharlampovich, A Myasnikov, Elementary theory of free non-abelian groups, J. Algebra 302 (2006) 451-552 MR2293770

[53] O Kharlampovich, A Myasnikov, On Tarski's decidability problem, preprint (2014) arXiv: 1402.0482

[54] O Kharlampovich, A Myasnikov, Decidability of the elementary theory of a torsionfree hyperbolic group, preprint (2015) arXiv:1303.0760

[55] H Kneser, Geschlossene Flächen in dreidimensionalen Mannigfaltigkeiten, Jahresber. Dtsch. Math.-Ver. 38 (1929) 248-260

[56] P Koiran, Hilbert's Nullstellensatz is in the polynomial hierarchy, J. Complexity 12 (1996) 273-286 MR1422712

[57] G Kuperberg, Knottedness is in NP, modulo GRH, Adv. Math. 256 (2014) 493-506 MR3177300 
[58] G Kuperberg, Algorithmic homeomorphism of 3-manifolds as a corollary of geometrization, preprint (2015) arXiv:1508.06720

[59] B Leeb, 3-manifolds with(out) metrics of nonpositive curvature, Invent. Math. 122 (1995) 277-289 MR1358977

[60] H Liang, Equation problem over central extensions of hyperbolic groups, J. Topol. Anal. 6 (2014) 167-192 MR3191648

[61] R C Lyndon, P E Schupp, Combinatorial group theory, Ergeb. Math. Grenzgeb. 89, Springer, Berlin (1977) MR0577064

[62] G S Makanin, Equations in a free group, Izv. Akad. Nauk SSSR Ser. Mat. 46 (1982) 1199-1273 MR682490 In Russian; translated in Math. USSR-Izv. 21 (1983) 546-582

[63] J Manning, Algorithmic detection and description of hyperbolic structures on closed 3-manifolds with solvable word problem, Geom. Topol. 6 (2002) 1-25 MR1885587

[64] D Marker, Model theory, Graduate Texts in Mathematics 217, Springer, New York (2002) MR1924282

[65] A Markov, The insolubility of the problem of homeomorphy, Dokl. Akad. Nauk SSSR 121 (1958) 218-220 MR0097793

[66] S Matveev, Algorithmic topology and classification of 3-manifolds, Algorithms and Computation in Mathematics 9, Springer, Berlin (2003) MR1997069

[67] J C C McKinsey, The decision problem for some classes of sentences without quantifiers, J. Symbolic Logic 8 (1943) 61-76 MR0008991

[68] K A Mihălova, The occurrence problem for direct products of groups, Dokl. Akad. Nauk SSSR 119 (1958) 1103-1105 MR0100018

[69] K A Mihaŭlova, The occurrence problem for free products of groups, Dokl. Akad. Nauk SSSR 127 (1959) 746-748 MR0106940

[70] K A Mihaŭlova, The occurrence problem for direct products of groups, Mat. Sb. 70 (112) (1966) 241-251 MR0194497

[71] K A Mihălova, The occurrence problem for free products of groups, Mat. Sb. 75 (117) (1968) 199-210 MR0222179

[72] C F Miller, III, Decision problems for groups - Survey and reflections, from: "Algorithms and classification in combinatorial group theory”, (G Baumslag, editor), Math. Sci. Res. Inst. Publ. 23, Springer, New York (1992) 1-59 MR1230627

[73] J Milnor, A unique decomposition theorem for 3-manifolds, Amer. J. Math. 84 (1962) 1-7 MR0142125

[74] A Minasyan, Hereditary conjugacy separability of right-angled Artin groups and its applications, Groups Geom. Dyn. 6 (2012) 335-388 MR2914863

[75] E E Moise, Affine structures in 3-manifolds, V: The triangulation theorem and Hauptvermutung, Ann. of Math. 56 (1952) 96-114 MR0048805 
[76] E E Moise, Geometric topology in dimensions 2 and 3, Graduate Texts in Mathematics 47, Springer, New York (1977) MR0488059

[77] J Morgan, G Tian, Ricci flow and the Poincaré conjecture, Clay Mathematics Monographs 3, Amer. Math. Soc. (2007) MR2334563

[78] J Morgan, G Tian, The geometrization conjecture, Clay Mathematics Monographs 5, Amer. Math. Soc. (2014) MR3186136

[79] A W Mostowski, On the decidability of some problems in special classes of groups, Fund. Math. 59 (1966) 123-135 MR0224693

[80] G A Niblo, Separability properties of free groups and surface groups, J. Pure Appl. Algebra 78 (1992) 77-84 MR1154898

[81] G A Niblo, D T Wise, Subgroup separability, knot groups and graph manifolds, Proc. Amer. Math. Soc. 129 (2001) 685-693 MR1707529

[82] PS Novikov, Unsolvability of the conjugacy problem in the theory of groups, Izv. Akad. Nauk SSSR. Ser. Mat. 18 (1954) 485-524 MR0075196

[83] PS Novikov, On the algorithmic insolvability of the word problem in group theory, AMS Transl. 9, Amer. Math. Soc. (1958) MR0075197

[84] P Papasoglu, On the sub-quadratic isoperimetric inequality, from: "Geometric group theory”, (R Charney, M Davis, M Shapiro, editors), Ohio State Univ. MSRI Publ. 3, de Gruyter, Berlin (1995) 149-157 MR1355100

[85] P Papasoglu, An algorithm detecting hyperbolicity, from: "Geometric and computational perspectives on infinite groups", (G Baumslag, D Epstein, R Gilman, H Short, C Sims, editors), DIMACS Ser. Discrete Math. Theoret. Comput. Sci. 25, Amer. Math. Soc. (1996) 193-200 MR1364185

[86] G Perelman, The entropy formula for the Ricci flow and its geometric applications, preprint (2002) arXiv:math/0211159

[87] G Perelman, Finite extinction time for the solutions to the Ricci flow on certain threemanifolds, preprint (2003) arXiv:math/0307245

[88] G Perelman, Ricci flow with surgery on three-manifolds, preprint (2003) arXiv: math/0303109

[89] B Poonen, Undecidable problems: A sampler, from: "Interpreting Gödel: Critical essays", (J Kennedy, editor), Cambridge Univ. Press (2014) 211-241

[90] J-P Préaux, Conjugacy problem in groups of oriented geometrizable 3-manifolds, Topology 45 (2006) 171-208 MR2170497

[91] J-P Préaux, The conjugacy problem in groups of non-orientable 3-manifolds, preprint (2013) arXiv:1202.4148

[92] P Przytycki, D T Wise, Separability of embedded surfaces in 3-manifolds, Compos. Math. 150 (2014) 1623-1630 MR3260144 
[93] MO Rabin, Recursive unsolvability of group theoretic problems, Ann. of Math. 67 (1958) 172-194 MR0110743

[94] K Reidemeister, Homotopieringe und Linsenräume, Abh. Math. Sem. Univ. Hamburg 11 (1935) 102-109 MR3069647

[95] E Rips, Z Sela, Canonical representatives and equations in hyperbolic groups, Invent. Math. 120 (1995) 489-512 MR1334482

[96] J H Rubinstein, An algorithm to recognize the 3-sphere, from: "Proc. ICM, Vol. 1, 2”, (S D Chatterji, editor), Birkhäuser, Basel (1995) 601-611 MR1403961

[97] S Schleimer, Sphere recognition lies in NP, from: "Low-dimensional and symplectic topology”, (M Usher, editor), Proc. Sympos. Pure Math. 82, Amer. Math. Soc. (2011) 183-213 MR2768660

[98] P Scott, Subgroups of surface groups are almost geometric, J. London Math. Soc. 17 (1978) 555-565 MR0494062

[99] P Scott, The geometries of 3-manifolds, Bull. London Math. Soc. 15 (1983) 401-487 MR705527

[100] P Scott, There are no fake Seifert fibre spaces with infinite $\pi_{1}$, Ann. of Math. 117 (1983) 35-70 MR683801

[101] P Scott, H Short, The homeomorphism problem for closed 3-manifolds, Algebr. Geom. Topol. 14 (2014) 2431-2444 MR3331689

[102] Z Sela, The conjugacy problem for knot groups, Topology 32 (1993) 363-369 MR1217075

[103] Z Sela, The isomorphism problem for hyperbolic groups, I, Ann. of Math. 141 (1995) 217-283 MR1324134

[104] Z Sela, A report on Tarski's decidability problem, preprint (2014) arXiv:1401.5711

[105] J-P Serre, Trees, Springer, Berlin (1980) MR607504

[106] C C Sims, Computation with finitely presented groups, Encycl. Math. Appl. 48, Cambridge Univ. Press (1994) MR1267733

[107] J Stallings, Grushko's theorem, II: Kneser's conjecture, Not. Amer. Math. Soc. 6 (1959) 531-532

[108] J Stillwell, The word problem and the isomorphism problem for groups, Bull. Amer. Math. Soc. 6 (1982) 33-56 MR634433

[109] A Thompson, Thin position and the recognition problem for $S^{3}$, Math. Res. Lett. 1 (1994) 613-630 MR1295555

[110] W P Thurston, Three-dimensional manifolds, Kleinian groups and hyperbolic geometry, Bull. Amer. Math. Soc. 6 (1982) 357-381 MR648524

[111] A M Turing, On computable numbers, with an application to the Entscheidungsproblem, Proc. London Math. Soc. S2-42 230 MR1577030 
[112] F Waldhausen, On irreducible 3-manifolds which are sufficiently large, Ann. of Math. 87 (1968) 56-88 MR0224099

[113] F Waldhausen, Recent results on sufficiently large 3-manifolds, from: "Algebraic and geometric topology, Part 2", (R J Milgram, editor), Proc. Sympos. Pure Math. 32, Amer. Math. Soc. (1978) 21-38 MR520520

[114] D T Wise, Research announcement: The structure of groups with a quasiconvex hierarchy, Electron. Res. Announc. Math. Sci. 16 (2009) 44-55 MR2558631

[115] D T Wise, From riches to raags: 3-manifolds, right-angled Artin groups, and cubical geometry, CBMS Reg. Conf. Ser. Math. 117, Amer. Math. Soc. (2012)

[116] D T Wise, The structure of groups with a quasi-convex hierarchy (2012) Available at http://tinyurl.com/wise-structure

University of California

Los Angeles, CA 90095, USA

Fakultät für Mathematik, Universität Regensburg

D-93053 Regensburg, Germany

Department of Pure Mathematics and Mathematical Statistics

University of Cambridge, Wilberforce Road, Cambridge CB3 OWB, UK

matthias@math.ucla.edu, sfriedl@gmail.com, h.wilton@maths.cam.ac.uk

Received: 16 February 2015 